\title{
Recent Developments in Electron Spin Science and Technology in Japan
}

\author{
Toshikazu Nakamura ${ }^{1} \cdot$ Tadaaki Ikoma $^{2} \cdot$ Ken-ichi Yamada ${ }^{3}$
}

Published online: 9 July 2018

(c) Springer-Verlag GmbH Austria, part of Springer Nature 2018

\begin{abstract}
Although general-purpose X-band electron spin resonance (ESR) techniques are well established, many advanced specialized methods have been developed for fields such as physics, chemistry, biology, pharmacy, and medicine. It should also be noted that the boundaries between such fields have become much less clearly defined than they previously were, leading to interdisciplinary research and advances in measurement techniques. This special issue of Applied Magnetic Resonance is devoted to recent developments in advanced ESR measurement techniques and interdisciplinary research of electron spin science and technology in Japan. The content of this issue is described below.

In the field of solid-state physics, it is often necessary to perform measurements at extremely low temperatures or under ultra-high magnetic fields, where significant quantum phenomena appear. In addition, in semiconductor research, it is sometimes desirable to investigate a device structure while applying an electric field. The progress of research into ESR measurements under such special external conditions is introduced.
\end{abstract}

Dynamic studies on processes, such as light-induced relaxation, diffusion in solutions, and chemical reactions, are also major topics of research in the field of chemistry. As new functional materials are being developed, the importance of such studies is expected to increase. We report advanced research into the use of ESR to investigate such systems.

Double electron-electron resonance (DEER or PELDOR) and electron nuclear double resonance (ENDOR) measurements are powerful experimental methods in

Toshikazu Nakamura

t-nk@ims.ac.jp

Tadaaki Ikoma

ikoma@chem.sc.niigata-u.ac.jp

Ken-ichi Yamada

kenyamada@phar.kyushu-u.ac.jp

1 Institute for Molecular Science, Okazaki, Japan

2 Niigata University, Niigata, Japan

3 Kyushu University, Fukuoka, Japan 
structural biology. A great deal of protein research has been performed in Japanese institutes using these techniques, and some of the research results are presented in this special issue. Recently, double electron-electron resonance has been applied to fields other than biology. One hot topic in solid-state physics is the charge distribution in semiconductor devices, and we introduce an example of double electron-electron resonance results in this area.

In the field of pathology, it is important to the improvement of methodology such as in vivo ESR imaging and dynamic nuclear polarization (DNP). There is also a need to develop functional probes and sensitizers. Moreover, it is important to practice pathological analysis by these electron spin probe methods. It is essential that the above research is carried out simultaneously. In this issue, several cases of practical research on medical applications of advanced ESR techniques in Japan are reported.

In summary, ESR-related techniques offer a great deal of potential in all the fields described above. It is also noted that interdisciplinary research communities are rapidly growing in Japan. We would like this type of cooperation to become firmly established and the results obtained to be disseminated worldwide.

We hope that this special issue will stimulate new developments by researchers already working in these areas, and also act as a trigger for those who are not familiar with ESR. We would like to thank all of the authors who contributed to this issue. We extend special thanks to Dr. Kev Salikhov, the Editor in Chief, who give us the opportunity to present this recent progress. 